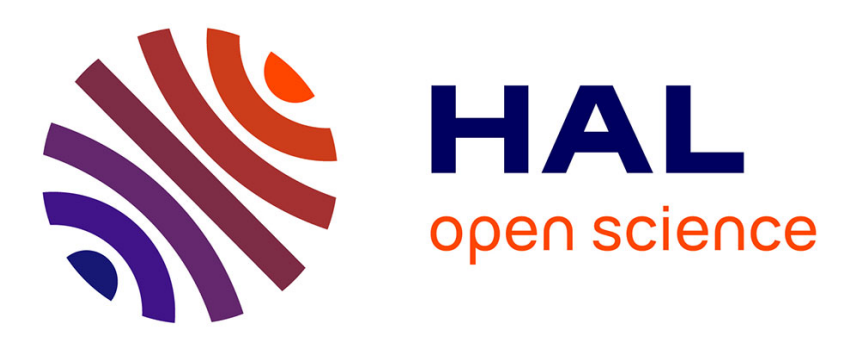

\title{
Vector Lyapunov Function based Stability for a Class of Impulsive Systems
}

Héctor Ríos, Laurentiu Hetel, Denis Efimov

\section{To cite this version:}

Héctor Ríos, Laurentiu Hetel, Denis Efimov. Vector Lyapunov Function based Stability for a Class of Impulsive Systems. 54th IEEE Conference on Decision and Control (CDC), 2015, Dec 2015, Osaka, Japan. hal-01218955

\section{HAL Id: hal-01218955 \\ https://hal.inria.fr/hal-01218955}

Submitted on 21 Oct 2015

HAL is a multi-disciplinary open access archive for the deposit and dissemination of scientific research documents, whether they are published or not. The documents may come from teaching and research institutions in France or abroad, or from public or private research centers.
L'archive ouverte pluridisciplinaire $\mathbf{H A L}$, est destinée au dépôt et à la diffusion de documents scientifiques de niveau recherche, publiés ou non, émanant des établissements d'enseignement et de recherche français ou étrangers, des laboratoires publics ou privés. 


\title{
Vector Lyapunov Function based Stability for a Class of Impulsive Systems
}

\author{
Héctor Ríos ${ }^{\dagger}$, Laurentiu Hetel ${ }^{\ddagger}$ and Denis Efimov ${ }^{\dagger}$
}

\begin{abstract}
This paper contributes to the exponential stability analysis for impulsive dynamical systems based on a vector Lyapunov function and its divergence operator. The method relies on a $2 D$ time domain representation. The results are applied to analyze the exponential stability of linear impulsive systems based on LMIs. Some examples illustrate the feasibility of the proposed approach.
\end{abstract}

Index Terms-Impulsive systems, Exponential stability.

\section{INTRODUCTION}

I MPULSIVE systems [1] represent an important class of hybrid systems [5] containing discontinuities or jumps in the trajectories of the system governed by discrete dynamics. There exist a large variety of phenomena that are characterized by abrupt changes in the system state at certain instants, e.g. power electronics, sample-data systems, models in economics, bursting rhythm models in medicine, etc. According to the manner impulses are triggered several types of impulsive systems can be distinguishable: systems with time dependent impulses, systems with state dependent impulses (reset systems), and the combination of both of them (see, e.g. [1], [5] and [14]). In the current work impulsive systems with time triggered impulses are studied.

In the context of stability analysis of impulsive systems an important effort has been made for linear dynamics. In [2], a functional-based approach is developed for stability analysis. This method introduces looped functional leading to LMI conditions which allows to establish dwell-time results. Exponential stability, based on Lyapunov functions with discontinuity at the impulses, is proposed by [9] for nonlinear time-varying impulsive systems but the stability conditions are only applied to the linear case. In the sampledata system framework, there exist many works where the impulsive systems are used to describe the behavior of aperiodic sample-data systems (see, e.g. [6] and [12]). These approaches rely on the equivalent correspondence between continuous and discrete time domains showed in [11]. It is worth mentioning that there exist some works related to the stability for nonlinear $2 D$ systems based on vector Lyapunov

\footnotetext{
${ }^{\dagger}$ Non-A team @ Inria, Parc Scientifique de la Haute Borne, 40 avenue Halley, 59650 Villeneuve d'Ascq, France; and CRIStAL (UMR-CNRS 9189), Ecole Centrale de Lille, BP 48, Cité Scientifique, 59651 Villeneuved'Ascq, France. Emails: hector.rios_barajaseinria.fr; denis.efimoveinria.fr

${ }^{\ddagger}$ CRIStAL (UMR-CNRS 9189), Ecole Centrale de Lille, BP 48, Cité Scientifique, 59651 Villeneuve-d'Ascq, France. Email: laurentiu.heteldec-lille.fr
}

function approach, i.e. [3] and [4]. However, those results are not directly applicable for impulsive systems.

In this paper, based on a $2 D$ time domain equivalence (see, e.g. [10] and [13]), the exponential stability notion for a class of impulsive systems is studied using a vector Lyapunov function approach [7]. The proposed method contributes to the development of a stability analysis method for impulsive dynamical systems based on a divergence operator of the vector Lyapunov function in a $2 D$ time domain. The main result is applied to analyze the exponential stability of linear impulsive systems based on LMIs. Some numerical examples illustrate the feasibility of the proposed approach.

The outline of this work is as follows. The problem statement and a preliminary definition of exponential stability are given in Section II. The main result as well as the conditions of exponential stability are described in Section III. Application of the developed theory to the problem of exponential stability for linear impulsive systems is considered in Section IV. Finally, some concluding remarks are discussed in Section V.

\section{Problem Statement}

Consider the class of impulsive dynamical systems for which the impulse times are external to the system and time dependent, i.e.

$$
\begin{aligned}
& \dot{x}(t)=f_{1}(x(t)), \quad \forall t \in \mathbb{R}_{+} \backslash \mathbb{I}, \\
& x(t)=f_{2}\left(x\left(t^{-}\right)\right), \quad \forall t \in \mathbb{I}, \\
& x(0)=x_{0},
\end{aligned}
$$

where $x, x_{0} \in \mathbb{R}^{n}$ are the state of the system and the initial condition, respectively. The set of impulse times $\mathbb{I}:=\left\{t_{i}\right\}_{i \in \mathbb{N}}$ is a countable subset of $\mathbb{R}_{+}$with $t_{i+1}-t_{i}>0$, for all $i \in \mathbb{N}$ and $t_{0}=0$ and $\lim _{i \rightarrow \infty} t_{i}=+\infty$ in order to avoid any zeno phenomena. The state trajectory is assumed to be right continuous and to have left limits at all times. The notation $x\left(t_{i+1}^{-}\right)$denotes the left limit of $x(t)$ as $t$ goes to $t_{i+1}$ from the left, i.e. $x\left(t^{-}\right)=\lim _{t \uparrow t_{i+1}} x(t)$. The distance between the impulses, i.e. the dwell-time (see, e.g. [8] for more details), is defined as $T_{i}:=t_{i+1}-t_{i} \leq T_{\max }$, for all $i \in \mathbb{N}$. The Lipschitz nonlinear functions $f_{1}$ and $f_{2}$ are such that $f_{1}(0)=0$ and $f_{2}(0)=0$. It is assumed that for all $x_{0} \in \mathbb{R}^{n}, f_{1}$ is such that system (1) has a unique solution over any time interval $\left[t_{i}, t_{i+1}\right)$ for all $i \in \mathbb{N}$.

The proposed Vector Lyapunov function based approach relies on the embedding of system (1)-(2) into a $2 D$ time 
domain. Indeed, the entire state trajectory $x(t)$ can be viewed as a sequence of the diagonal dynamics ${ }^{1}$ of the following $2 D$ system:

$$
\begin{gathered}
\frac{d x}{d t}(t, k)=f_{1}(x(t, k)), \quad \forall t \in\left[t_{i}, t_{i+1}\right), \\
x(t, k+1)=f_{2}(x(t, k)), \quad \forall t=t_{i+1}, \\
\forall i=k \in \mathbb{N}, t_{0}=0, x(0,0)=x_{0}^{0},
\end{gathered}
$$

where $x(t, k):\left[t_{i}, t_{i+1}\right) \times \mathbb{N} \rightarrow \mathbb{R}^{n}$ is the current state vector, $x\left(t_{i+1}, k+1\right): t_{i+1} \times \mathbb{N} \rightarrow \mathbb{R}^{n}$ represents the reset vector state, $x\left(t_{i+1}, k\right): t_{i+1} \times \mathbb{N} \rightarrow \mathbb{R}^{n}$ denotes the value of $x$ just before the switching $k+1$, for all $i=k \in \mathbb{N}$, i.e. $x\left(t_{i+1}, k\right)=$ $\lim _{t \uparrow t_{i+1}} x(t, k)$, for $t<t_{i+1}$. It is worth saying that the solutions of system (3) are unique for the diagonal dynamics, i.e. for all $i=k \in \mathbb{N}, t \in \mathbb{R}_{+}$; and they are represented by a sequence of functions $\left\{x\left(t_{i}+\tau, k\right), \tau \in\left(0, t_{i}+1\right]\right\}_{i}$, for all $i=k \in \mathbb{N}$.

Assume that $|q|$ denotes the Euclidean norm of a vector $q$. The following stability definitions are introduced:

Definition 1. A $2 D$ system described by (3)-(4), is said to be diagonal exponentially stable (DES) if there exist positive constants $\kappa_{1}, \kappa_{2}, c$ and $\varepsilon$ such that

$$
\begin{gathered}
\left|x\left(t_{i+1}, k+1\right)\right|^{2} \leq c \kappa_{1}^{k+1}\left|x_{0}^{0}\right|^{2}, 0<\kappa_{1}<1-\varepsilon, \\
|x(t, k)|^{2} \leq \kappa_{2}\left|x\left(t_{i}, k\right)\right|^{2}, \forall t \in\left[t_{i}, t_{i+1}\right),
\end{gathered}
$$

for all $i, k \in \mathbb{N}$, and a small positive $\varepsilon$.

The goal in this work is to find the conditions for the stability of the impulsive systems described by (1)-(2) by means of the $2 D$ time representation (3)-(4).

\section{Main Result: Stability of Impulsive Systems}

In order to give the stability conditions a vector Lyapunov approach is used, i.e.

$$
V\left(t, x(t, k), x\left(t_{i+1}, k+1\right)\right)=\left[\begin{array}{c}
V_{1}(t, x(t, k)) \\
V_{2}\left(x\left(t_{i+1}, k+1\right)\right)
\end{array}\right],
$$

where $V_{1}(t, x)>0, V_{2}(x)>0$, for all $t \geq 0$ and $x \neq 0$, and $V_{1}(t, 0)=0, V_{2}(0)=0, \forall t \geq 0$. Now, the divergence operator of the candidate function $V$ along the trajectories of system (3)-(4) is introduced for all $t \in\left[t_{i}, t_{i+1}\right)$ :

$$
\begin{array}{r}
\operatorname{div} V\left(t, x(t, k), x\left(t_{i+1}, k+1\right)\right)=\frac{d V_{1}(t, x(t, k))}{d t} \\
+V_{2}\left(x\left(t_{i+1}, k+1\right)\right)-V_{2}\left(x\left(t_{i+1}, k\right)\right),
\end{array}
$$

i.e. $V_{1}$ is differentiable with respect to continuous time $t$ while the difference in $V_{2}$ is calculated in discrete time. Based on the previous explanations, the following theorem is established:

Theorem 1. Assume that for some $T_{i}$ there exist positive constants $\varepsilon, c_{1}, c_{2}, c_{3}, c_{4}$ and $c_{5}$ such that the vector

\footnotetext{
${ }^{1}$ The diagonal dynamics make reference only to those dynamics given by (3)-(4) corresponding to $i=k$, for all $i, k \in \mathbb{N}$ and for all $t \in \mathbb{R}^{+}$.
}

Lyapunov function $V\left(t, x(t, k), x\left(t_{i+1}, k+1\right)\right)$ and its divergence along the trajectories of the system (3)-(4) satisfy, for all $t \in\left[t_{i}, t_{i+1}\right)$, the following inequalities:

$$
\begin{gathered}
c_{1}|x(t, k)|^{2} \leq V_{1}(t, x(t, k)) \leq c_{2}|x(t, k)|^{2}, \\
c_{3}\left|x\left(t_{i+1}, k\right)\right|^{2} \leq V_{2}\left(x\left(t_{i+1}, k\right)\right) \leq c_{4}\left|x\left(t_{i+1}, k\right)\right|^{2}, \\
\operatorname{div} V \leq-c_{5}\left(|x(t, k)|^{2}+\left|x\left(t_{i+1}, k\right)\right|^{2}\right), \\
c_{2}\left(c_{4}-c_{5}\right) \leq c_{1} c_{5} \vee T_{i}<\frac{c_{2}}{c_{5}} \alpha, \\
\frac{c_{2}}{c_{5}} \gamma<T_{i},
\end{gathered}
$$

where $\gamma=-\ln \left[\frac{c_{3}(1-\varepsilon)}{c_{2}}\right]$ and $\alpha=-\ln \left[\frac{c_{2}\left(c_{4}-c_{5}\right)-c_{1} c_{5}}{c_{2}\left(c_{4}-c_{5}\right)}\right]$ for all $c_{2}\left(c_{4}-c_{5}\right)>c_{1} c_{5}$. Then, the $2 D$ system (3)-(4) is DES.

Proof. It is omitted due to lack of space.

The result given by Theorem 1 can be perfectly depicted by the following algorithm:

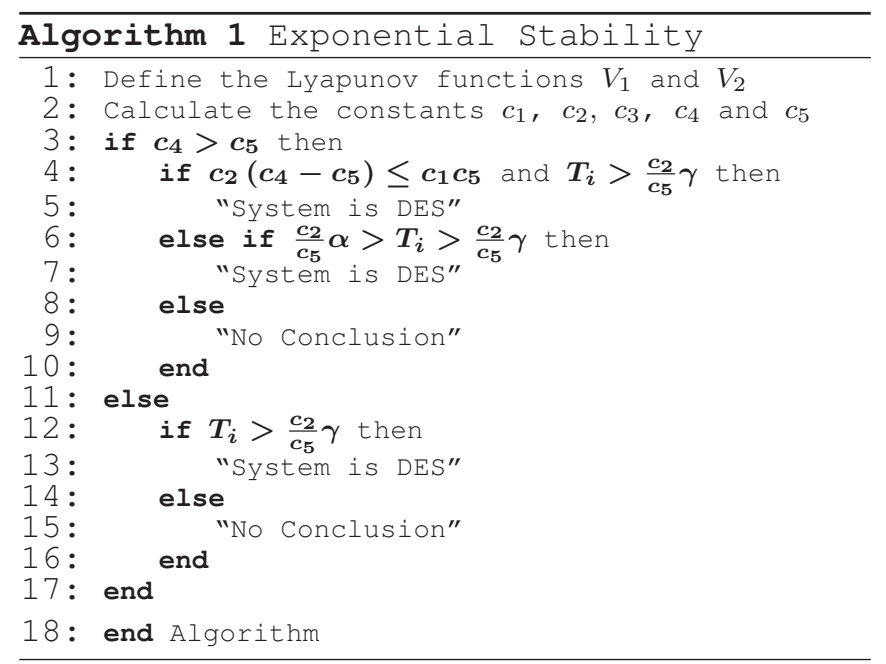

Remark 1. Based on the statements given by Theorem 1, the Algorithm 1 provides some notions of minimum and maximum dwell-time depending on the structure of the system dynamics. In particular, the first and third case for diagonal exponential stability (pseudo-code lines: 5 and 13, Algorithm 1) give conditions for minimum dwell-time while the second case (pseudo-code lines: 7, Algorithm 1) provides conditions for maximum dwell-time.

In the following, the statements given by Theorem 1 are applied to prove the exponentially stability of linear impulsive systems.

\section{Exponential Stability of Linear ImpUlsive SYSTEMS}

Consider the following class of linear impulsive systems

$$
\begin{gathered}
\frac{d x}{d t}(t, k)=A x(t, k), \quad \forall t \in\left[t_{i}, t_{i+1}\right), \\
x(t, k+1)=E x(t, k), \quad \forall t=t_{i+1}, \\
\forall i=k \in \mathbb{N}, t_{0}=0, x(0,0)=x_{0}^{0},
\end{gathered}
$$


where $A$ and $E$ are constant matrices with corresponding dimensions. It is assumed that $A$ is Hurwitz and $E$ is Schur. Then, the direct application of Theorem 1, provide us the following corollary that establishes the conditions to ensure the diagonal exponentially stability, described by Definition 1 , for the linear impulsive system (14)-(15).

Corollary 1. Consider the vector Lyapunov function $V\left(x(t, k), x\left(t_{i+1}, k+1\right)\right)$ in (7), with $V_{1}(x(t, k))=$ $x^{T}(t, k) P_{1} x(t, k)$ and $V_{2}\left(x\left(t_{i+1}, k+1\right)\right)=x^{T}\left(t_{i+1}, k+\right.$ 1) $P_{2} x\left(t_{i+1}, k+1\right)$. If there exist matrices $P_{1}=P_{1}^{T}>0$ and $P_{2}=P_{2}^{T}>0$, such that

$$
\left[\begin{array}{cc}
P_{1} A+A^{T} P_{1} & 0 \\
0 & E^{T} P_{2} E-P_{2}
\end{array}\right]=-Q
$$

for $Q=Q^{T}>0$, and the constraints (12) and (13) hold; for $c_{1}=\lambda_{\min }\left(P_{1}\right), c_{2}=\lambda_{\max }\left(P_{1}\right), c_{3}=\lambda_{\min }\left(P_{2}\right)$, $c_{4}=\lambda_{\max }\left(P_{2}\right)$ and $c_{5}=\lambda_{\min }(Q)$; then, the linear impulsive system (14)-(15) is DES. ${ }^{2}$

Proof. It is clear that the vector Lyapunov function $V\left(x(t, k), x\left(t_{i+1}, k+1\right)\right)$ satisfies inequalities (9) and (10), i.e.

$$
\begin{gathered}
\lambda_{\min }\left(P_{1}\right)|x(t, k)|^{2} \leq V_{1}(x(t, k)) \leq \lambda_{\max }\left(P_{1}\right)|x(t, k)|^{2}, \\
\lambda_{\min }\left(P_{2}\right)\left|x\left(t_{i+1}, k\right)\right|^{2} \leq V_{2}\left(x\left(t_{i+1}, k\right)\right) \leq \lambda_{\max }\left(P_{2}\right)\left|x\left(t_{i+1}, k\right)\right|^{2} .
\end{gathered}
$$

From the divergence definition and the previous inequalities, it is obtained that for all $t \in\left[t_{i}, t_{i+1}\right)$

$$
\begin{aligned}
\operatorname{div} V=x^{T}(t, k)\left[P_{1} A+A^{T} P_{1}\right] x(t, k) & \\
+x^{T}\left(t_{i+1}, k+1\right) & P_{2} x\left(t_{i+1}, k+1\right) \\
& -x^{T}\left(t_{i+1}, k\right) P_{2} x\left(t_{i+1}, k\right),
\end{aligned}
$$

and using the reset equation (15), it follows that

$$
\begin{aligned}
\operatorname{div} V= & x^{T}(t, k)\left[P_{1} A+A^{T} P_{1}\right] x(t, k) \\
& +x^{T}\left(t_{i+1}, k\right)\left[E^{T} P_{2} E-P_{2}\right] x\left(t_{i+1}, k\right) .
\end{aligned}
$$

From the matrix equality (16), it is obtained

$$
\operatorname{div} V \leq-\lambda_{\min }(Q)\left(|x(t, k)|^{2}+\left|x\left(t_{i+1}, k\right)\right|^{2}\right) .
$$

Then, the divergence of $V$ satisfies the inequality (11), and according to the statements given by Theorem 1, system (14)-(15) is DES, if the constraints (12) and (13) hold.

Example 1. Let us consider system (14)-(15) with

$$
A=\left[\begin{array}{cc}
-1 & 0 \\
1 & -2
\end{array}\right], \quad E=\left[\begin{array}{cc}
0.5 & 0 \\
0 & 0.5
\end{array}\right] .
$$

Note that both matrices are stable, i.e. $A$ is Hurwitz and $E$ is Schur. Then, Corollary 1 is applied and the following feasible results are obtained:

$$
\begin{gathered}
P_{1}=\left[\begin{array}{ll}
5.2500 & 0.7500 \\
0.7500 & 2.2500
\end{array}\right], \quad P_{2}=12 I_{2}, \\
Q=9 I_{4},
\end{gathered}
$$

\footnotetext{
${ }^{2}$ The values of $\lambda_{\min }(Y)$ and $\lambda_{\max }(Y)$ correspond to the minimum and maximum eigenvalues of a matrix $Y$, respectively.
}

with $c_{1}=2.0729, c_{2}=5.4271, c_{3}=12, c_{4}=12$ and $c_{5}=9$. Therefore, according to the Algorithm 1, as it was expected, the impulsive system is DES for all $T_{i}>0$ (It is easy to check that $c_{4}>c_{5}$ and $c_{2}\left(c_{4}-c_{5}\right) \leq c_{1} c_{5}$ hold and then the first case for diagonal exponential stability is obtained). The trajectories of the system, for $T_{i}=0.5$, are depicted in Fig. 1.

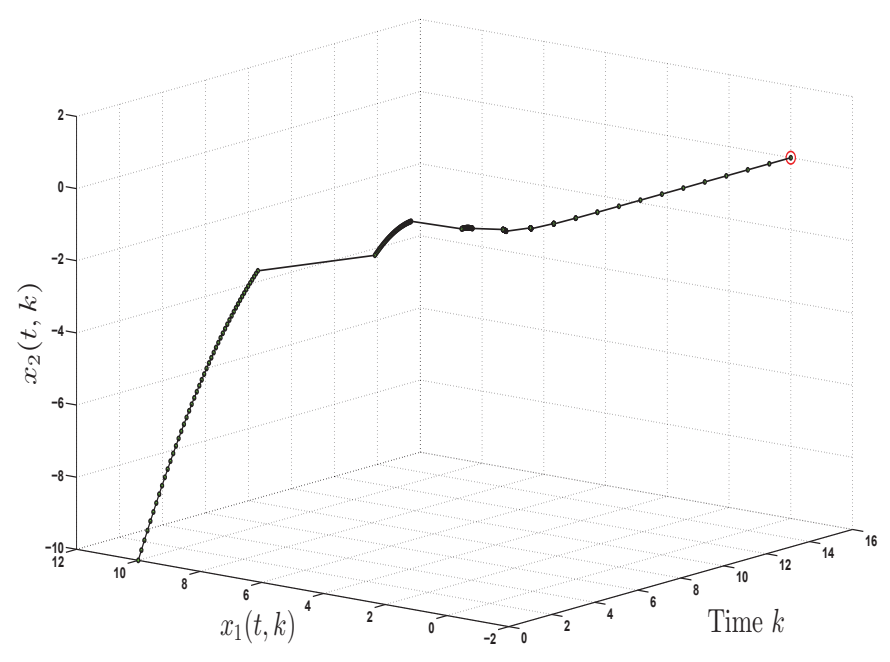

Figure 1. Example 1 (Stable-Stable): Trajectories of the linear impulsive system for $T_{i}=0.5$.

In order to deal with linear impulsive systems where matrix $A$, or matrix $E$, is not Hurwitz, or not Schur, respectively; it is necessary to improve the structure of the Lyapunov function $V\left(t, x(t, k), x\left(t_{i+1}, k+1\right)\right)$, to be precisely $V_{1}(t, x(t, k))$. In this sense, the following corollaries provide different ways to design $V_{1}(t, x(t, k))$ and the conditions to get diagonal exponential stability.

Corollary 2. Consider the vector Lyapunov function $V\left(t, x(t, k), x\left(t_{i+1}, k+1\right)\right) \quad$ in (7), with $V_{2}\left(x\left(t_{i+1}, k+1\right)\right)=x^{T}\left(t_{i+1}, k+1\right) P_{2} x\left(t_{i+1}, k+1\right)$, and

$$
\begin{gathered}
V_{1}(t, x(t, k))=x^{T}(t, k) P(t) x(t, k), \\
P(t)=P 1+\Delta(t)\left[R-e^{A^{T} \Delta(t)} E^{T} R E e^{A \Delta(t)}\right],
\end{gathered}
$$

where $\Delta(t)=T_{i}-\tau(t)$, with $\dot{\tau}(t)=1$, and $\tau\left(t_{i}\right)=0$, $\forall t \in\left[t_{i}, t_{i+1}\right)$. Then, if there exist matrices $R=R^{T}>0$, $P_{1}=P_{1}^{T}>0$ and $P_{2}=P_{2}^{T}>0$, such that

$$
\begin{gathered}
\Upsilon_{0}+T_{i} \Upsilon_{1}=-Q_{1}, \\
\Upsilon_{0}=-Q_{2},
\end{gathered}
$$

for $Q_{1}=Q_{1}^{T}>0$ and $Q_{2}=Q_{2}^{T}>0$, with

$$
\begin{gathered}
\Upsilon_{0}=\left[\begin{array}{cc}
P_{1} A+A^{T} P_{1}-R & R \\
\star & E^{T} P_{2} E-P_{2}-E^{T} R E
\end{array}\right], \\
\Upsilon_{1}=\left[\begin{array}{cc}
R A+A^{T} R & -A^{T} R E \\
\star & 0
\end{array}\right],
\end{gathered}
$$


and the constraints (12) and (13) hold; for $c_{1}=$ $\lambda_{\min }\left(P_{1}\right), \quad c_{2}=\lambda_{\max }\left(P_{1}\right)+T_{i} \lambda_{\max }(R)\left(1+\varphi^{2}\right)$, with $\varphi=\sup _{s \in\left[0, T_{i}\right]} \lambda_{\max }\left(E e^{A \Delta(s)}\right), \quad c_{3}=\lambda_{\min }\left(P_{2}\right), c_{4}=$ $\lambda_{\max }\left(P_{2}\right)$ and $c_{5}=\min \left(\lambda_{\min }\left(Q_{1}\right), \lambda_{\min }\left(Q_{2}\right)\right)$; then, the linear impulsive system (14)-(15) is DES.

Proof. First, taking into account that $x\left(t_{i+1}, k+1\right)=$ $E e^{A \Delta(t)} x(t, k), V_{1}(t, x(t, k))$ can be rewritten as follows

$$
\begin{array}{rl}
V_{1}(t, x(t, k))=x^{T}(t, k) P_{1} & x(t, k) \\
& +\Delta(t) \xi^{T}(t, k) R \xi(t, x),
\end{array}
$$

where $\xi(t, x)=x(t, k)-x\left(t_{i+1}, k+1\right)$. Now, from the divergence definition it is obtained that for all $t \in\left[t_{i}, t_{i+1}\right)$

$$
\begin{aligned}
\operatorname{div} V= & x^{T}(t, k)\left[P_{1} A+A^{T} P_{1}\right] x(t, k) \\
& +\Delta(t) \xi^{T}(t, k) R \frac{d \xi(t, x)}{d t} \\
+ & {\left[\begin{array}{c}
\left.\Delta(t) \frac{d \xi^{T}(t, x)}{d t}-\xi^{T}(t, k)\right] R \xi(t, x) \\
+
\end{array}\right.} \\
& +x^{T}\left(t_{i+1}, k\right)\left[E^{T} P_{2} E-P_{2}\right] x\left(t_{i+1}, k\right)
\end{aligned}
$$

$$
\begin{aligned}
\operatorname{div} V= & x^{T}(t, k)\left[P_{1} A+A^{T} P_{1}\right] x(t, k) \\
& +\Delta(t) \xi^{T}(t, k) R A x(t, k) \\
& +\left[\Delta(t) x^{T}(t, k) A^{T}-\xi^{T}(t, k)\right] R \xi(t, x) \\
& +x^{T}\left(t_{i+1}, k\right)\left[E^{T} P_{2} E-P_{2}\right] x\left(t_{i+1}, k\right) .
\end{aligned}
$$

Then, it follows that

$$
\begin{aligned}
\operatorname{div} V \leq\left[\begin{array}{ll}
x^{T}(t, k) & x^{T}\left(t_{i+1}, k\right)
\end{array}\right] \times & {\left[\begin{array}{l}
\Upsilon_{0}+\left(T_{i}-\tau(t)\right) \Upsilon_{1}
\end{array}\right] \times\left[\begin{array}{c}
x(t, k) \\
x\left(t_{i+1}, k\right)
\end{array}\right] . }
\end{aligned}
$$

Since this LMI is affine in $\tau$, its negative definiteness over the interval $\left[t_{i}, t_{i+1}\right)$ is given by the negative definiteness at the vertices of the set, i.e. over the finite set $\tau \in\left\{0, T_{i}\right\}$; hence, the LMIs (19) and (20) are provided. Then, if these LMIs are feasible, the divergence of $V$ satisfies (11) with $c_{5}=\min \left(\lambda_{\min }\left(Q_{1}\right), \lambda_{\min }\left(Q_{2}\right)\right)$.

Finally, in order to take advantage of Theorem 1, it is necessary to prove that $V_{1}(t, x(t, k))$ satisfies inequality (9). From definition of $V_{1}(t, x(t, k))$, it follows that

$$
\begin{aligned}
V_{1}(t, x(t, k)) \leq & \lambda_{\max }\left(P_{1}\right)|x(t, k)|^{2}+T_{i} \lambda_{\max }(R)|\xi(t, k)|^{2}, \\
\leq & \lambda_{\max }\left(P_{1}\right)|x(t, k)|^{2} \\
& +T_{i} \lambda_{\max }(R)\left(|x(t, k)|^{2}+\left|E x\left(t_{i+1}, k\right)\right|^{2}\right), \\
\leq & \lambda_{\max }\left(P_{1}\right)|x(t, k)|^{2} \\
& +T_{i} \lambda_{\max }(R)\left(|x(t, k)|^{2}+\left|E e^{A \Delta(t)} x(t, k)\right|^{2}\right), \\
\leq & {\left[\lambda_{\max }\left(P_{1}\right)+T_{i} \lambda_{\max }(R)\left(1+\varphi^{2}\right)\right]|x(t, k)|^{2}, }
\end{aligned}
$$

where $\varphi=\sup _{s \in\left[0, T_{i}\right]} \lambda_{\max }\left(E e^{A \Delta(s)}\right)$, thus; the upper bound is obtained. For the lower bound, it holds that $V_{1}(t, x(t, k)) \geq \lambda_{\min }\left(P_{1}\right)|x(t, k)|^{2}$. Therefore, the lower bound is also obtained, and according to the statements given by Theorem 1, system (14)-(15) is DES, if the constraints (12) and (13) hold.

Example 2. Let us consider system (14)-(15) with

$$
A=\left[\begin{array}{cc}
1 & 3 \\
-1 & 2
\end{array}\right], \quad E=\left[\begin{array}{cc}
0.5 & 0 \\
0 & 0.5
\end{array}\right] \text {. }
$$

Note that the continuous dynamics is unstable while the discrete one is stable, i.e. $A$ is not Hurwitz and $E$ is Schur. Corollary 1 is applied together with a bisection-like approach and the following feasible results are obtained:

$$
\begin{aligned}
& P_{1}=\left[\begin{array}{cc}
0.0049 & -0.0056 \\
-0.0056 & 0.0215
\end{array}\right], \quad P_{2}=\left[\begin{array}{cc}
3.1018 & -0.4709 \\
-0.4709 & 4.3250
\end{array}\right] \text {, } \\
& Q_{1}=\left[\begin{array}{cccc}
0.0294 & -0.0303 & -0.1427 & 0.0276 \\
\star & 0.1197 & 0.1392 & -0.3810 \\
\star & \star & 1.2074 & -0.2009 \\
\star & \star & \star & 1.7367
\end{array}\right] \text {, } \\
& Q_{2}=\left[\begin{array}{cccc}
0.1664 & -0.0853 & -0.1769 & 0.0972 \\
\star & 0.4332 & 0.0972 & -0.4594 \\
\star & \star & 1.2074 & -0.2009 \\
\star & \star & \star & 1.7367
\end{array}\right] \text {, } \\
& R=\left[\begin{array}{cc}
0.3538 & -0.1944 \\
-0.1944 & 0.9188
\end{array}\right],
\end{aligned}
$$

with $c_{1}=0.0032, c_{2}=0.7350, c_{3}=2.9415, c_{4}=4.4852$ and $c_{5}=0.0052$. Therefore, according to the Algorithm 1, the impulsive system is DES for all $0.2499>T_{i}>0$ (It is easy to check that $c_{4}>c_{5}$ and $c_{2}\left(c_{4}-c_{5}\right)>c_{1} c_{5}$ hold and then the second case for diagonal exponential stability is obtained). The trajectories of the system, for $T_{i}=0.24$, are depicted in Fig. 2.

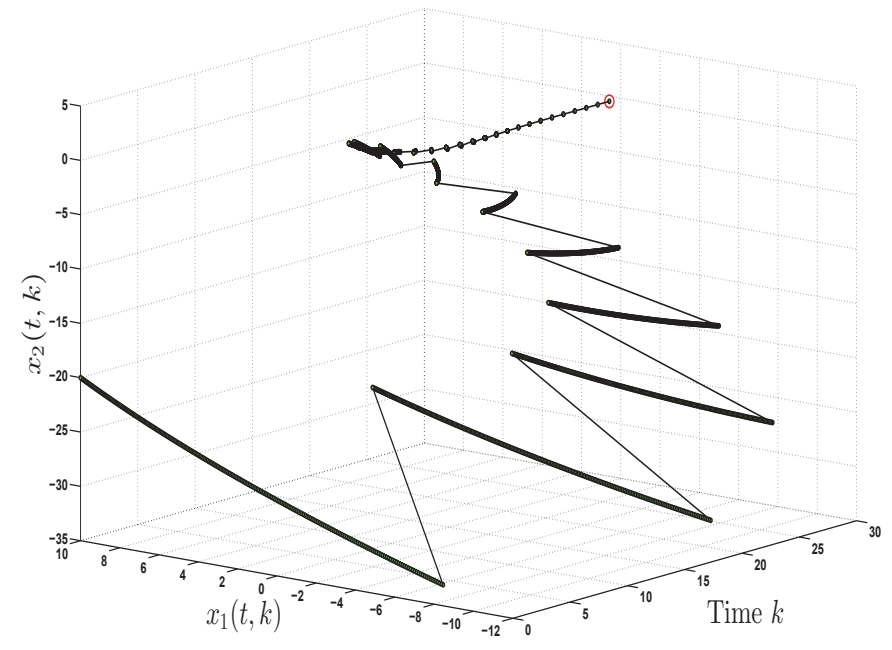

Figure 2. Example 2 (Unstable-Stable): Trajectories of the linear impulsive system for $T_{i}=0.24$.

Remark 2. Note that Example 2 (A unstable and E Schur) illustrates the conditions for maximum dwell-time given by Theorem 1 and depicted in pseudo-code lines: 7, Algorithm 1.

Example 3. Let us consider system (14)-(15) with

$$
A=\left[\begin{array}{cc}
-1 & 0 \\
1 & -2
\end{array}\right], \quad E=\left[\begin{array}{ll}
2 & 1 \\
1 & 3
\end{array}\right] \text {. }
$$


For this example the continuous dynamics is stable while the discrete one is unstable, i.e. $A$ is Hurwitz and $E$ is anti-Schur. Corollary 1 is applied and the following feasible results are obtained:

$$
\begin{gathered}
P_{1}=\left[\begin{array}{cc}
2.0271 & -0.0469 \\
-0.0469 & 0.8244
\end{array}\right], \quad P_{2}=0.2 I_{2}, \\
Q_{1}=3 I_{4}, \quad Q_{2}=3 I_{4} \\
R=\left[\begin{array}{cc}
1.8958 & -0.8244 \\
-0.8244 & 1.0176
\end{array}\right]
\end{gathered}
$$

with $c_{1}=0.8225, c_{2}=2.1564, c_{3}=0.2, c_{4}=0.2$ and $c_{5}=3$. Therefore, according to the Algorithm 1, the impulsive system is DES for all $T_{i}>1.7092$ (it is easy to check that $c_{5}>c_{4}$ holds and then the third case for diagonal exponential stability is obtained). The trajectories of the system, for $T_{i}=2$, are depicted in Fig. 3 .

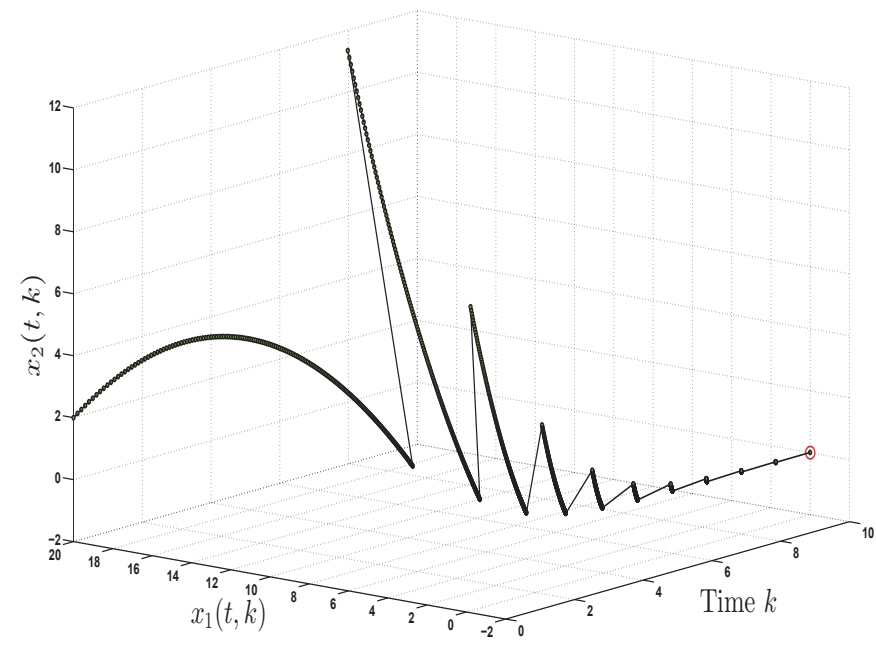

Figure 3. Example 3 (Stable-Unstable): Trajectories of the linear impulsive system for $T_{i}=0.2$.

Remark 3. Note that Example 3 (A Hurwitz and E antiSchur) illustrates the conditions for minimum dwell-time given by Theorem 1 and depicted in pseudo-code lines: 5 and 13, Algorithm 1.

\section{CONCLusions}

In this paper the exponential stability notion for a class of impulsive systems is studied using a vector Lyapunov function approach. The proposed method contributes to the development of a stability analysis for impulsive dynamical systems based on a vector Lyapunov function and its divergence operator in a $2 D$ time domain. The main result is applied to analyze the exponential stability of linear impulsive systems based on LMIs, and some numerical examples illustrate the feasibility of the proposed approach. The analysis of exponential stability of nonlinear systems is in the scope of the future research.

\section{ACKNOWLEDGMENT}

This work was supported in part by Conseil Region Nord-Pas de Calais (ESTIREZ), the Government of Russian Federation (Grant 074-U01), the Ministry of Education and Science of Russian Federation (Project 14.Z50.31.0031), and ANR ROCC-SYS (ANR-14-CE27-0008).

\section{REFERENCES}

[1] D. Bainov and P. Simeonov. Systems with Impulse Effects: Stability, Theory and Applications. Academy Press, 1989.

[2] C. Briat and A. Seuret. A looped-functional approach for robust stability of linear impulsive systems. Systems \& Control Letters, 61(10):980-988, 2012.

[3] M. Emelianov, P. Pakshin, K. Gałkowski, and E. Rogers. Stability and stabilization of differential nonlinear repetitive processes with applications. In In Proccedings of the 19th IFAC World Congress, pages 5467-5472, Cape Town, South Africa, 2014.

[4] J. Emelianova, P. Pakshin, K. Gałkowski, and E. Rogers. Vector lyapunov function based stability of a class of applications relevant $2 \mathrm{~d}$ nonlinear systems. In In Proccedings of the 19th IFAC World Congress, pages 8247-8252, Cape Town, South Africa, 2014.

[5] R. Goebel, R.G. Sanfelice, and A.R. Teel. Hybrid dynamical systems. IEEE Control Systems Magazine, 29(2):28-93, 2009.

[6] L. Hu, Y. Cao, and H. Shao. Constrained robust sampled-data control of nonlinear uncertain systems. International Journal of Robust and Nonlinear Control, 12:447-155, 2002.

[7] V. Lakshmikantham, V. M. Matrosov, and S. Sivasundaram. Vector Lyapunov Functions and Stability Analysis of Nonlinear Systems. Mathematics and its Applications. Kluwer Academic Publishers Group, Dordrecht, 1991.

[8] D. Liberzon. Switching in Systems and Control. Systems and Control: Foundations and Applications. Birkhäuser, Boston, MA, 2003.

[9] P. Naghshtabrizi, J.-P. Hespanha, and A.-R. Teel. Exponential stability of impulsive systems with application to uncertain sampled-data systems. Systems and Control Letters, 57(5):378-385, 2008.

[10] E. Rogers, K. Galkowski, and D. Owens. Control Systems Theory and Applications for Linear Repetitive Processes, volume 349 of Lecture Notes in Control and Information Sciences. Springer-Verlag, Berlin, 2007.

[11] A. Seuret. A novel stability analysis of linear systems under asynchronous samplings. Automatica, 48(1):177-182, 2012.

[12] N. Sivashankar and P.P. Khargonekar. Characterization of the $\mathcal{L}_{2}$ induced norm for linear systems with jumps with applications to sampled-data systems. SIAM Journal on Control and Optimization, 32(4):1128-1150, 1994

[13] N. Yeganefar, N. Yeganefar, M. Ghamgui, and E. Moulay. Lyapunov theory for 2-d nonlinear roesser models: Application to asymptotic and exponential stability. IEEE Transactions on Automatic Control, 58(5):1299-1304, 2013.

[14] S.T. Zavalishchin and A.N. Sesekin. Dynamic Impulse Systems. Theory and Applications. Mathematics and its Applications. Kluwer Academic Publishers Group, Dordrecht, 1997. 